\title{
Psychological Factors in Medical Disorders Assessed with the Diagnostic Criteria for Psychosomatic Research
}

\author{
Piero Porcelli ${ }^{a}$ Orlando Todarellob \\ aPsychosomatic Unit, IRCCS De Bellis Hospital, Castellana Grotte, and bepartment of Psychiatry, \\ University of Bari, Bari, Italy
}

\begin{abstract}
The Diagnostic Criteria for Psychosomatic Research (DCPR) are a diagnostic and conceptual framework that aims to translate psychosocial variables that derived from psychosomatic research into operational tools whereby individual patients could be identified. A set of 12 syndromes was developed and grouped in the clusters of abnormal illness behavior (health anxiety, disease phobia, thanatophobia, and illness denial), somatization (functional somatic symptoms secondary to a psychiatric disorder, persistent somatization, conversion symptoms, and anniversary reaction), irritability (type A behavior, irritable mood), demoralization, and alexithymia. This chapter aims to illustrate the criteria for each of the 12 DCPR syndromes and how to diagnose them with the aid of the DCPR Structured Interview, as illustrated in a clinical example.

Copyright $\odot 2012$ S. Karger AG, Basel
\end{abstract}

The DSM-IV criteria for diagnosing somatoform disorders have attracted increasing and considerable criticism for several reasons, including the failure to represent adequately clinical reality, the restatement of the dualistic dichotomy between medical illnesses and psychosocial syndromes, the tendency to overpsychologize somatic symptoms (when axis I disorders are present) or to underestimate psychological aspects (when medical diagnoses are established), and the neglect of subsyndromal conditions, personality and behavioral factors $[1,2]$. To the purpose of identifying patients within a given illness population whose psychological factors have a relevant relative weight of clinical significance, in the last decade an international group of investigators proposed the Diagnostic Criteria for Psychosomatic Research (DCPR) [3] that include 12 clusters of clinically important psychosomatic correlates of medical disorders. This paper aims to illustrate how to diagnose the DCPR syndromes by means of the DCPR Structured Interview (DCPR-SI). 
The diagnostic criteria for each of the 12 DCPR syndromes are shown in online supplementary appendix A. The DCPR system has undergone extensive validation in the last 10 years from research studies recently summarized in a monograph [4] and two reviews $[2,5]$. The DCPR system enables clinicians to identify psychological and subclinical problems in medical patients to a much higher extent than the DSM classification since patients diagnosed with DCPR but not DSM syndromes were 3.6 times more prevalent than patients with DSM but not DCPR diagnoses. Furthermore, it has been able to identify patients with poorer psychosocial functioning and maladaptive illness-related coping in several medical settings (dermatology, oncology, endocrinology, and consultation psychiatry) and to predict treatment outcome in gastroenterology and higher vulnerability for coronary heart disease in postmyocardial infarction $[2,4,5]$.

\section{The Diagnostic Criteria for Psychosomatic Research-Structured Interview}

The DCPR categories listed in appendix A are assessed through the DCPR-SI (see online suppl. appendix B) which has shown good to excellent characteristics of reliability and validity. Used by trained investigators, the interview has resulted in substantial interrater agreement for all 12 syndromes (Cohen's $\mathrm{k}$ ranging from 0.69 to 0.97) [6]. Construct-related validity has been evaluated with sound external criteria by independent studies with different medical samples showing overall correct classification rate of $70-77 \%$ for alexithymia [7-9] and $61-73 \%$ for type A behavior (TAB) [9].

The DCPR-SI is administered during a face-to-face interview and takes about 15-30 min to be completed. It includes 58 items scored in a yes/no response format evaluating the presence of 1 or more of the 12 DCPR syndromes over the past 6 or 12 months. The interview requires the assessor's knowledge of the patient's main and comorbid medical diagnoses, clinical judgment, information on the course of the medical disorder, and familiarity with the DCPR literature. Psychiatric assessment is not strictly needed but is however suggested in conjunction with the DCPR-SI. Most questions are asked directly to the patient, while some items are to be answered by the interviewer because of the need of extra-interview information, such as the chronology of symptom onset for functional somatic symptoms secondary to a psychiatric disorder and the patient's extraverbal behavior for conversion disorder, TAB, and alexithymia. Items investigating these last aspects are italicized in the DCPR-SI form. Depending on the purpose of the interview (specific medical setting or disorder, or research hypotheses), the DCPR-SI might be administered in part by excluding selected DCPR categories. The duration of the interview may therefore vary. The main characteristic of each syndrome is inquired first, and if the criterion is not met, the interviewer has to skip to the following syndrome. In online supplementary appendix C, a clinical vignette is used as an example for scoring the DCPR-SI and reaching diagnostic conclusions. 
In the following description of DCPR clusters, letters and numbers for criteria refer to the DCPR-SI (appendix B).

\section{Abnormal Illness Behavior}

The first 4 DCPR clusters (disease phobia, thanatophobia, health anxiety, and illness denial) are related to patients' ways of perceiving, experiencing, evaluating, and responding to their health status that are subsumed into the construct of abnormal illness behavior (AIB) [10] and are issued from Kellner's Illness Attitude Scale [11].

Health anxiety's main feature is the unfounded fear of having a severe disease even without experiencing specific somatic symptoms (A1), as in hypochondriasis, and/or the presence of common somatic symptoms (A2) - two features that are thought to relate to somatosensory amplification. If the patient does not meet these 2 characteristics, the interviewer has to skip to the following 'disease phobia'. Unlike hypochondriasis, worries and fears readily respond to appropriate reassurance (B), even though new worries may ensue after some time, and are short-lived (less than 6 months' duration; C). High prevalence of health anxiety has been found mostly in oncology (38\%), consultation-liaison (CL) psychiatry (35\%), and frequent attenders in primary care $(28 \%)$, even though a substantial high proportion of about $10 \%$ was found in cardiology, gastroenterology, and dermatology [4]. Psychological treatments focused on explaining to the patient the mechanisms that link emotional distress to selective perceptions of somatic symptoms in a self-perpetuating vicious circle can be suggested for patients with health anxiety [12].

Disease phobia is defined by the experience of severe anxiety, even in the form of panic attack, of suffering from a specific disease (A), for instance AIDS or cancer, unlike the unfounded fear of a having whatever serious disease in the previous 'health anxiety'. Additionally, the patient reports that he or she has been provided with documentable medical reassurance (B) and that the phobic symptoms have lasted for at least 6 months (C). Disease phobia can be distinguished from hypochondriasis because of the phobic quality of fears (acute in disease phobia, constant in hypochondriasis) and the nature of the phobic object (stable over time in disease phobia, changeable in hypochondriasis). The differential diagnosis between hypochondriasis and disease phobia is relevant for treatment planning. The phobic quality of symptoms typically leads to avoidance, and may therefore be treated with in vitro or in vivo exposure strategies, while hypochondriacal patients do not respond to exposure because they tend to exhibit 'doctor-shopping behavior' rather than avoidance. In a sense, disease phobia is related to hypochondriasis as panic disorder is to generalized anxiety. Consistently, the prevalence of the DCPR category of disease phobia is very high in frequent attenders in primary care (34\%) and trivial in a community sample (1\%) [4]. 
Thanatophobia is defined by the main characteristic of a sense of impending death or even the firm belief of dying soon, without a real reason, interfering significantly with the patient's level of functioning (A). Differently from health anxiety and disease phobia that are focused on the fear of having a serious disease, thanatophobia is characterized by the frightened anxiety of death and the accompanying avoidant behavior focused on any news related to death (e.g. funerals and obituary notices; B) and any situation related to death, as changing TV channel, interrupting conversations concerning death people or disasters or accidents (criterion C). It is not frequent (prevalence of $0.6 \%$ in a community sample and $1.5-3 \%$ in clinical settings), even though it is present in a relevant proportion in patients with specific illnesses as myocardial infarction (7\%) and cancer (11\%), and who make high use of health care services (12\%) [4].

Illness denial is placed at the opposite pole with respect to hypochondriasis within the AIB conceptual framework, and is characterized mainly by the minimization, or even conscious failure, of symptom reporting to the physician (A1) and the lack of adherence to appropriate medical advices regarding treatment or other recommendations (A2). Also, the patient has to be fully aware of his or her health condition as it would result from adequate medical explanation about diagnosis and treatment recommendations (B). The high prevalence of this DCPR category in primary care (up to $80 \%$ ) and CL psychiatry (29\%) suggests it might hide under the appearance of 'normality' or lead to hospitalization because of behaviors that foster doubts in the physicians. Denying the burden of physical disease might be an adaptive coping mechanism in some circumstances and at certain degrees, as in the early stage after diagnosis or in the terminal phase of a life-threatening disease because it may alleviate psychological distress. However, within the AIB framework, denying, distorting or minimizing clinical relevance, personal responsibility, long-term prognosis, and need for treatment may have serious health-related consequences [4].

\section{Somatization}

The four DCPR clusters of functional somatic symptoms secondary to psychiatric disorders, persistent somatization, conversion symptoms, and anniversary reaction are related to Lipowski's concept of somatization [13].

Functional somatic symptoms secondary to a psychiatric disorder are defined mainly by the lifetime experience of common somatic symptoms (e.g. palpitations, sweating, tremor, becoming flushed, gastrointestinal symptoms, dizziness, muscular pains, persistent tiredness) that often go unnoticed in most people or do not cause any consequence other than minor self-medication. In these patients, however, experiencing these symptoms greatly interferes with everyday life and prompts for repeated medical treatments (A). In the DCPR-SI, the 'no' answer to this item (that skips the interviews to 'persistent somatization') does not imply that the patient has never 
had these symptoms but that he or she considers them as so common that are not taken into consideration when talking about his or her overall health condition. If the patient's attention paid to these symptoms is high, the interviewer has to check whether the physician has considered them as medically unexplained (B). The interviewer has also to note whether a psychiatric disorder has been diagnosed previously or the patient suffered from some defined form of psychopathology before $(\mathrm{C})$ : the diagnosis is placed if the onset of psychopathology occurred prior to the onset of the functional somatic symptoms (D). This DCPR category is very useful in clinical practice because in the DSM the diagnosis of a somatoform condition is excluded by the presence of another axis I disorder (hierarchical rule), while it is very likely that a patient with psychopathology (major depression, panic disorder) has also clinically relevant somatic symptoms requiring additional specific treatment. The prevalence of functional somatic symptoms secondary to psychiatric disorders has been found in the range of $30-45 \%$ in high health care users, medical inpatients referred for psychiatric consultation, and functional gastrointestinal disorders in which there is also a high proportion (75-90\%) of DSM-IV disorders [5]. However, confirming the criticism toward the hierarchical rule [14], only one third of patients with DSM-IV mood and anxiety disorder were also diagnosed with functional somatic symptoms secondary to psychiatric disorders [4].

Persistent somatization is issued from Kellner's concept of symptom clustering [15] that highlights the fact that an individual with a psychosomatic condition (e.g. irritable bowel syndrome) is more likely to subsequently get another functional gastrointestinal (e.g. non-ulcer dyspepsia) or extragastrointestinal (e.g. chronic fatigue) syndrome over time. Persistent somatization is described by the main feature of a chronic ( $>6$ months) somatic functional disorder causing psychosocial problem and medical care seeking (A). Moreover, the somatic disorder has to be medically unexplained (B) and the patient reports that, after the prescribed medical treatments, he or she experienced exaggerated side effects $(\mathrm{C} 1)$ or even a worsening of his or her health status (C2), and/or additional symptoms of autonomic arousal (C3), suggesting a predisposition to chronic somatization, somatic amplification, lower pain threshold, and high suggestibility. The prevalence of persistent somatization was considerable (about $30 \%$ ) in gastroenterology and CL psychiatry and in patients with comorbid DSM-IV mood, anxiety, and adjustment disorders [4]. It is noteworthy that $82 \%$ of patients meeting criteria for DCPR somatization clusters did not satisfy the criteria for any DSM-IV somatoform disorder [16].

Conversion symptoms is issued by Engel's criteria [17] and is defined mainly by symptoms or deficits affecting voluntary sensory-motor functioning as balance problems, localized paralysis or weakness, loss of voice, eating difficulty, double vision or loss of sight (A). These symptoms have not been explained by specific medical causes after appropriate examinations (B). Unlikely, the DSM-IV conversion disorder that requires the exclusion of another somatoform disorder or a medical condition and is differentiated from somatization disorder because of the number of symptoms, 
this DCPR category requires at least 2 additional features related to the patient's past experience and personality features. The symptoms have to be preceded by some specific stressful events connected to symptoms (C1), already experienced in the past and/or observed in someone else close to patient (C2). Furthermore, the interviewer should notice if the patient shows a sort of ambivalence (the belle indifference often described in literature) in symptom reporting (e.g. the patient appears overtly relaxed or unconcerned while describing highly distressing symptoms; C3) and/or some features of histrionic personality (exhibitionist or inappropriately seductive behavior, seeking of reassurance or approval, exaggerated displays of emotions, high sensitivity to criticism or disapproval, excessive concern with physical appearance, a need to be the center of attention, low tolerance for frustration or delayed gratification, rapid shifting of emotional states, making rash decisions, self-centeredness). Consistently with the notion that false-positive cases of conversion symptoms are likely to be present in medical settings with a symptom-based only diagnosis neglecting the patient's psychological characteristics $[18,19]$, the DCPR category of conversion symptoms has been found in a proportion of 5-7\% in gastroenterology and cardiology [4].

Anniversary reaction was described by Engel [20], and is often unrecognized because of the lack of awareness of the patient and the negligence of the clinician. The main characteristic is given by the fact that the patient, upon the interviewer's request, pays his or her attention to some specific events that occurred at a given time before the onset of somatic symptoms (A). The following questions of the interview focus therefore on further details: if symptoms occur at an important date in the patient's life (e.g. birthday, Christmas) or started when the patient had the same age that a family member developed a life-threatening disease (e.g. abdominal pain starting when the patient was aged 52, which was her sister's age when she developed the first symptoms of intestinal cancer 10 years ago; B) or a serious health problem (chest pain at the age of 41 when the patient's brother had his first heart attack) or died (e.g. dysuria at the age of 64 when the patient's father died because of prostate cancer). Anniversary reactions may be more frequent than believed in PTSD war veterans [21] and were found in more than $10 \%$ of patients in primary care and oncology [4].

\section{Irritability}

Type A Behavior (TAB) is part of the irritability cluster along with irritable mood. TAB has become a classic construct in psychosomatic medicine and indicates a 'specific emotion-action complex' of individuals aggressively committed to struggle for achieving more and more in less and less time [22]. As a complex, several characteristics are included in TAB, and they are enlisted in question A of the DCPR-SI (at least 5 of them are necessary for diagnosing TAB at the DCPR-SI): a strong commitment to work because of excessive responsibility (A1); a strong sense of ambition and need for achievement and approval (A8); a sense of time urgency for several activities (either at 
work or not) that must be accomplished as soon as possible (A2); hostility and cynicism that may become aggressiveness when the patient needs to take others into consideration in pursuing a goal, particularly when he or she feels a strong sense of time urgency (e.g. driving fast, impatience at a traffic light stop; A4), and/or is evident in a strong sense of competitiveness (A9); speech and bodily movements (hands, arms, facial muscles, glances) characterized by tension, rapidity, fast pacing, and sometimes explosiveness, as shown at the interview (A3) and/or self-reported (A6); a general irritable attitude in coping with everyday life (A5); the feeling that mind is crowded with many ideas and thoughts, all important and all at the same time (A7). Clinical interest for TAB in medical patients requires however that the patient reports also somatic symptoms of autonomic arousal (palpitations, sweating, muscular and stomach pains, intestinal disorders, and/or breathing fast; B). Even though it is classically associated with cardiovascular disorders, the DCPR category of TAB was found highly frequent not only in cardiology (28\%) but also in frequent attenders in primary care (52\%), eating disorders (27\%), CL psychiatry (25\%) and also healthy people (25\%), suggesting it might be considered as a relevant psychosomatic factor across a variety of clinical and preclinical conditions requiring a careful evaluation from clinicians [4].

Irritable mood is issued from Snaith and Taylor's [23] description of a feeling state of irritability which may be experienced as brief episodes or it may be prolonged and generalized, requiring an increased effort to control, while overt manifestations lack the cathartic effect of justified outbursts of anger, thus being always unpleasant for the subject. The main criteria of the DCPR-SI require that the subject is aware of his or her negative feeling state, even though he or she is unable to have full control over it (A1) and/or experiences verbal or behavioral outbursts of rage (A2; ego-dystonic condition). Furthermore, the patient reports that aggressive behavior lacks the usual cathartic effect, thus leaving him or her with a sense of uneasiness (B) and with acute symptoms of autonomic arousal as tachycardia (C). Because of the direct or mediating role played by irritability in several medical conditions and predisposing unhealthy behaviors, the DCPR irritable mood has been found to be frequent (about $15 \%$ ) in all medical settings (particularly in patients with endocrinology illness, high health care use, and eating disorders) to the same extent as community subjects [4], and, although related, is independent of depression [24].

\section{Demoralization}

It is issued from Frank's [25] suggestion that demoralization results from the awareness of being unable to cope with a pressing problem or of having failed one's own or other's expectations, and is the main reason why subjects seek psychotherapeutic treatment. Subjective incompetence is considered as the clinical hallmark of demoralization and of related feelings of hopelessness and helplessness [26]. Indeed, the main DCPR criteria of demoralization include the sense of personal failure to meet 
his or her or others' expectations (A1), a subjective feeling of incompetence to cope with urgent problems (A2), and perception of hopelessness and helplessness (A3). Additional aspects are the prolonged period of demoralized states lasting for longer than one month (B) and the patient's perception that his or her feeling state is related to his or her health status (C). The clinical relevance of demoralization in physical syndromes is highlighted by its high prevalence in all medical settings and low frequency in the community sample (3\%) [4]. Demoralization and major depression, although overlapping, are distinct phenomena [27]: a depressed person is incapable of experiencing enjoyment of any sort because of a primary reduction in motivation and drive, whereas a demoralized individual is unable to acknowledge anticipatory pleasure because of inhibition in his or her initiative, but consummatory pleasure is unaffected [28].

\section{Alexithymia}

Like other DCPR syndromes, alexithymia is a classic theme in psychosomatic medicine. It is now recognized to be a multifaceted construct including two high-order factors (lack of affect awareness and operatory thinking) leading to a reduced ability to cognitively process feelings, and is considered one of the vulnerability factors for the development of medical and psychiatric disorders of affect regulation [29]. Some of the facets of the construct are inquired directly in the DCPR-SI, and at least 3 of them are necessary for the DCPR diagnosis of alexithymia, while some others (particularly the overall level of affect expression and communication) have to be inferred through clinical observation. Two direct questions concern the subject's ability to verbalize (A1) and to communicate emotional states (A2); two further questions are related to cognitive features as reduced ability to fantasizing (A3) and external-related thinking (A4); one further question concerns the manifestation of somatic problems (e.g. pain, sickness) subsequent to the experience of strong emotions (A5), and one last question concerns emotional, non-mentalized outbursts (e.g. sudden crying when the patient experiences a strong emotion, but he or she is unaware of the connection or not able to link; A6). The construct validity of the DCPR criteria of alexithymia has been confirmed in 3 independent studies [7-9]. As expected, a high rate of alexithymia was found in several medical settings such as oncology (26\%), functional gastrointestinal disorders (48\%), frequent attending patients in primary care (38\%), CL psychiatry (25\%), and eating disorders (27\%) [4].

\section{Conclusion}

A large body of psychosomatic literature has shown definitely that psychosocial factors, psychopathology, and somatic symptoms are intertwined at multiple levels and 
that the concept of illness is multifactorial in itself [30]. One of the main criticisms against the use of the traditional psychiatric classification with medical patients is the misleading assumption of the organic versus functional dichotomy claiming that the presence of an organic cause, as well as a hierarchical higher-order psychiatric disorder such as major depression or panic disorder, subsumes psychological disturbances and, vice versa, the absence of an organic cause strongly indicates the presence of a psychological or psychiatric reason. The development of the DCPR system focused on the task to translate psychological characteristics widely observed and studied in various medical settings into diagnostic criteria, which may entail clinical values and may be investigated through the reliable DCPR-SI across disorders, regardless of their presumed origin. By replacing the DSM-IV hierarchical rule with the concepts of association and coexistence of psychological, functional, and organic illnesses, not surprisingly the DCPR assessment was found to be more suitable than psychiatric criteria in identifying AIB, somatization, irritability, demoralization, and alexithymia in patients with a variety of medical disorders $[2,4,5]$. The accumulated evidence has led to the suggestion of replacing the DSM-IV category of 'psychological factors affecting medical condition' - a poorly defined diagnosis with little, if any, impact on clinical practice - with a new section including hypochondriasis and the most frequent DCPR syndromes [31,32].

The application of DCPR through the structured interview presented in this paper might hopefully allow a more specific designation of problems that commonly present to both primary care as well as psychiatric physicians and a more accurate identification of psychosomatic distress across different somatic disorders (whether of functional or organic in nature). As DCPR reflects the need of adopting a global psychosomatic approach to health, the use of the DCPR-SI offers a unique comprehensive tool for eliciting meaningful clinical information that cannot be obtained otherwise and allows health care providers (general practitioners, medical specialists, psychiatrists, clinical psychologists, psychotherapists, psychosomatic specialists) to bring together a large number of seemingly unrelated disorders whose names have been scattered so far under the headings of the various anatomical systems and to pave the ground for multidisciplinary work in clinical medicine [33].

\section{References}

1 Wise TN, Birket-Smith TN: The somatoform disorders for DSM-V: the need for change in process and contents. Psychosomatics 2000;43:437-440.

-2 Sirri L, Fabbri S, Fava GA, Sonino N: New strategies in the assessment of psychological factors affecting medical conditions. J Person Ass 2007;89:216-228.
3 Fava GA, Freyberger HJ, Bech P, Christodoulou G, Sensky T, Theorell T, Wise TN: Diagnostic criteria for use in psychosomatic research. Psychother Psychosom 1995;63:1-8.

4 Porcelli P, Sonino N (eds): Psychological Factors Affecting Medical Conditions. A New Classification for DSM-V. Adv Psychosom Med. Basel, Karger, 2007, vol 28. 
5 Porcelli P, Rafanelli C: Criteria for Psychosomatic Research (DCPR) in the medical setting. Curr Psychiatry Rep 2010;12:246-254.

6 Galeazzi GM, Ferrari S, Mackinnon A, Rigatelli M: Interrater reliability, prevalence, and relation to ICD-10 diagnoses of the Diagnostic Criteria for Psychosomatic Research in consultation-liaison psychiatry patients. Psychosomatics 2004;45:386393.

7 Fukunishi I, Hosaka T, Aoki T, Azekawa T, Ota A, Miyaoka H: Criterion-related validity of diagnostic criteria for alexithymia in a general hospital psychiatric setting. Psychother Psychosom 1996;65:82-85.

8 Porcelli P, De Carne M: Criterion-related validity of the Diagnostic Criteria for Psychosomatic Research for alexithymia in patients with functional gastrointestinal disorders. Psychother Psychosom 2001;70: 184-188.

\9 Beresnevaité M, Taylor GJ, Bagby RM: Assessing alexithymia and Type A behavior in coronary heart disease patients: a multimethod approach. Psychother Psychosom 2007;76:186-192.

10 Pilowsky I: Abnormal Illness Behavior. Chichester, Wiley, 1997.

11 Sirri L, Grandi S, Fava GA: The illness attitude scales. Psychother Psychosom 2008;77:337-350.

- 12 Fava GA, Grandi S, Rafanelli C, Fabbri S, Cazzaro M: Explanatory therapy in hypochondriasis. J Clin Psychiatry 2000;61:317-322.

13 Lipowski ZJ: Somatization. Am J Psychiatry 1987; 47:160-167.

14 Fava GA, Sonino N: The biopsychosocial model thirty years later. Psychother Psychosom 2008;77: $1-2$.

15 Kellner R: Psychosomatic syndromes, somatization and somatoform disorders. Psychother Psychosom 1994;61:4-24.

- 16 Mangelli L, Bravi A, Fava GA, Ottolini F, Porcelli P, Rafanelli C, Rigatelli M, Sonino N: Assessing somatization with various diagnostic criteria. Psychosomatics 2009;50:38-41.

17 Engel GL: Conversion symptoms; in Mac Bryde CM, Blacklow RS (eds): Signs and Symptoms. Philadelphia, Lippincott, 1970, pp 650-669.

18 Stone J, Smyth R, Carson A, Lewis S, Prescott R, Warlow C, Sharpe M: Systematic review of misdiagnosis of conversion symptoms and 'hysteria. BMJ 2005;331:989.
9 Stonnington CM, Barry JJ, Fisher RS: Conversion disorder. Am J Psychiatry 2006;163:1510-1517.

20 Engel GL: The death of a twin: mourning and anniversary reactions. Fragments of 10 years of selfanalysis. Int J Psychoanal 1975;56:23-40.

21 Morgan CA, Hill S, Fox P, Kingham P, Southwick SM: Anniversary reactions in Gulf War veterans: a follow-up inquiry 6 years after the war. Am J Psychiatry 1999;156:1075-1079.

22 Friedman M, Rosenman RH: Type A Behavior and Your Heart. New York, Knopf, 1974.

23 Snaith RP, Taylor CM: Irritability. Br J Psychiatry 1985;147:127-136.

24 Mangelli L, Fava GA, Grassi L, Ottolini F, Paolini S, Porcelli P, Rafanelli C, Rigatelli M, Sonino N: Irritable mood in Italian patients with medical disease. J Nerv Ment Dis 2006;194:226-228.

25 Frank JD: Psychotherapy: the restoration of morale. Am J Psychiatry 1974; 131:271-274.

26 Cockram CA, Doros G, de Figueiredo JM: Diagnosis and measurement of subjective incompetence: the clinical hallmark of demoralization. Psychother Psychosom 2009;78:342-345.

27 Mangelli, L, Fava GA, Grandi S, Grassi L, Ottolini F, Porcelli P, Rafanelli C, Rigatelli M, Sonino N: Assessing demoralization and depression in the setting of medical disease. J Clin Psychiatry 2005;66:391-394.

28 Clarke DM, Kissane DW: Demoralization: its phenomenology and importance. Aust N Z J Psychiatry 2002;36:733-742.

29 Bagby RM, Taylor GJ, Parker JD, Doclems SE: The development of the Toronto Structured Interview for Alexithymia: item selection, factor structure, reliability and concurrent validity. Psychother Psychosom 2006;75:25-39.

30 Fava GA, Sonino N: The clinical domains of psychosomatics medicine. J Clin Psychiatry 2005;66: 849-858.

31 Fava GA, Fabbri S, Sirri L, Wise TN: Psychological factors affecting medical condition: a new proposal for DSM-V. Psychosomatics 2007;48:103-111.

2 Fava GA, Wise TN: Psychological factors affecting either identified or feared medical conditions: a solution for somatoform disorders. Am J Psychiatry 2007;164:1002-1003.

33 Fava GA, Sonino N: Psychosomatic assessment. Psychother Psychosom 2009;78:333-341.

\author{
Dr. Piero Porcelli \\ Psychosomatic Unit, IRCCS De Bellis Hospital \\ Via Turi 27 \\ IT-70013 Castellana Grotte (Italy) \\ Tel. +39 080 4994685, E-Mail porcellip@media.it
}

